\title{
Dissociations of the number and precision of visual short-term memory representations in change detection
}

\author{
Weizhen Xie $^{1}$ - Weiwei Zhang ${ }^{1}$
}

Published online: 24 July 2017

(C) Psychonomic Society, Inc. 2017

\begin{abstract}
The present study dissociated the number (i.e., quantity) and precision (i.e., quality) of visual short-term memory (STM) representations in change detection using receiver operating characteristic (ROC) and experimental manipulations. Across three experiments, participants performed both recognition and recall tests of visual STM using the change-detection task and the continuous color-wheel recall task, respectively. Experiment 1 demonstrated that the estimates of the number and precision of visual STM representations based on the ROC model of change-detection performance were robustly correlated with the corresponding estimates based on the mixture model of continuous-recall performance. Experiments 2 and 3 showed that the experimental manipulation of mnemonic precision using white-noise masking and the experimental manipulation of the number of encoded STM representations using consolidation masking produced selective effects on the corresponding measures of mnemonic precision and the number of encoded STM representations, respectively, in both change-detection and continuous-recall tasks. Altogether, using the individualdifferences (Experiment 1) and experimental dissociation (Experiment 2 and 3) approaches, the present study demonstrated the some-or-none nature of visual STM representations across recall and recognition.
\end{abstract}

Electronic supplementary material The online version of this article (doi:10.3758/s13421-017-0739-7) contains supplementary material, which is available to authorized users.

Weiwei Zhang

weiwei.zhang@ucr.edu

1 Department of Psychology, University of California, Riverside, 900 University Ave., Riverside, CA 92521, USA
Keywords Visual short-term memory · Masking · Capacity · Precision $\cdot$ ROC

When it comes to memory, both quantity and quality matter (Koriat \& Goldsmith, 1996; Koriat, Goldsmith, \& Pansky, 2000). The quantitative aspect of memory refers to the likelihood that a given stimulus is encoded into and retrieved from the mental storehouse. The qualitative aspect of memory refers to the correspondence between the external stimuli and the internal memory representations, which can be operationalized as the inverse of the variability of memory performance. Although it is theoretically important to distinguish these two aspects of memory representations (Koriat \& Goldsmith, 1996; Zhang \& Luck, 2011), it is only until recently that these two aspects of memory have been simultaneously modeled in a single task to address theoretical questions (Bays \& Husain, 2008; Brady, Konkle, Gill, Oliva, \& Alvarez, 2013; Wilken \& Ma, 2004; Zhang \& Luck, 2008).

The qualitative and quantitative aspects of memory representations can be mathematically modeled based on their independent contributions to overall memory recall performance in a delayed estimation task (Zhang \& Luck, 2008, 2009, 2011). In this task, observers memorize some briefly presented memory items, such as colored squares, and then recall a given memory item after a delay of seconds (e.g., Zhang \& Luck, 2008) or minutes (e.g., Brady et al., 2013), by reproducing the color of the memory item in a continuous manner. The overall recall performance across trials reflects mixed contributions of two components (and hence the mixture model), namely the noisy internal memory and random responses due to failed memory retrieval (Zhang \& Luck, 2008). Using standard model-fitting procedures, two parameters can be estimated from the overall recall data, yielding estimates of a quantitative aspect and a qualitative aspect of retrieved memory 
representations. Specifically, the quantitative measure is the probability of successful memory retrieval, operationalized as the probability of random responses due to failed memory retrieval subtracted from one. The qualitative measure is mnemonic precision of retrieved memory, which is inversely related to the variability of memory recall performance.

This mixture model (Zhang \& Luck, 2008, 2009, 2011) can distinguish the quantitative and qualitative aspects of memory representations and processes, including memory encoding (Zhang \& Luck, 2008) and forgetting (Zhang \& Luck, 2009). It can also be used to distinguish experimental effects on the quantitative and qualitative of memory, such as memory impairments across clinical populations (Gold et al., 2010), and effects of sociobiological factors on memory, including sleep problems (Wee, Asplund, \& Chee, 2013), emotion (Spachtholz, Kuhbandner, \& Pekrun, 2014; Xie \& Zhang, 2016, 2017c), and aging (Peich, Husain, \& Bays, 2013). Using this approach, some previous effects that have been attributed to the qualitative aspect of memory could instead result from changes in the quantitative aspect of memory (Zhang \& Luck, 2008; 2009; 2011). For example, the gradual increase in the overall variability of memory performance in visual short-term memory (STM) across retention intervals could be better accounted for by declines in the likelihood of successful memory retrieval (Chris Donkin, Nosofsky, Gold, \& Shiffrin, 2015) than by declines in the quality of memory representation (Cornelissen \& Greenlee, 2000; Lee \& Harris, 1996; Paivio \& Bleasdale, 1974). More importantly, this approach can also be used to address theoretically significant questions, such as whether STM storage is constrained by a quantitative limit (i.e., capacity; Zhang \& Luck, 2008), a qualitative limit (i.e., precision; Bays \& Husain, 2008; van den Berg, Shin, Chou, George, \& Ma, 2012), or both (Alvarez \& Cavanagh, 2004).

Notwithstanding these important applications in addressing empirical and theoretical issues, the mixture model initially developed to account for recall data (Zhang \& Luck, 2008, 2009,2011 ) has three constraints that limit its application in a broader context. First, it is unclear whether the mixture model can be generalized from recall to recognition, because recall and recognition may be supported by different retrieval processes (e.g., remembering vs. knowing; Mandler, 1980; Tulving, 1985; but see Haist, Shimamura, \& Squire, 1992; Wixted, 2007) or representations (Smith \& Nielsen, 1970). Given the wider applications of recognition than recall in the literature, partially due to the reduced task difficulty in recognition (Bernbach \& Kupchak, 1972; Hollingworth, 1913) and the dependence of memory sensitivity on testing procedures (Makovski, Watson, Koutstaal, \& Jiang, 2010), it is important to generalize the mixture model of memory from recall to recognition. Second, the mixture model for recall tasks requires participants to continuously estimate or reproduce a feature of the remembered stimuli, limiting this model to stimuli with reproducible features (e.g., color and orientation). Third, the mixture model has some assumptions that may only be valid under limited circumstances. For example, when memory representations cannot be successfully retrieved from memory, recall responses are assumed to be random guesses (e.g., randomly distributed across trials). This assumption is more likely to be valid for features that continuously vary in a circular space (e.g., color and orientation) than features in a line space (e.g., contrast and size) or in a limited range of a circular space (e.g., orientations within certain degrees of angles). The latter could exhibit systematic biases such as responses toward the categorical features (Hardman, Vergauwe, \& Ricker, 2017; Huttenlocher, Hedges, \& Duncan, 1991), making it difficult to model random responses due to failed memory retrieval. These categorical responses also violate an additional assumption that feature representations are homogeneous across the entire feature space (Bae, Olkkonen, Allred, Wilson, \& Flombaum, 2014).

The present study thus investigated whether the previously established mixture model of recall (Zhang \& Luck, 2008) can be generalized to recognition within the domain of visual STM, the temporary memory buffer that has a limited capacity to actively maintain visual memory representations over a short delay interval (Cowan, 2001). A widely used recognition task in visual STM is the change-detection task (Luck \& Vogel, 1997; Phillips, 1974). In this task participants memorize several stimuli that are briefly presented in a memory array. After a short blank delay period, a test array appears with one stimulus at the location of an original memory item (single-probe procedure; Rouder, Morey, Morey, \& Cowan, 2011). This stimulus is equally likely to be the same as or different from the original memory item presented at the same location in the memory array. Participants report the presence or absence of a change in the test array as accurately as possible. In this task, the number of retained memory representations in visual STM can be estimated using Cowan's K or Pashler's K computed from hit rate and false alarm rate (Cowan, 2001; Luck \& Vogel, 1997; Rouder et al., 2011). However, it is less straightforward to estimate mnemonic precision of retained STM representations using this paradigm. An indirect method to infer STM precision is based on differences in change-detection performance when the dissimilarity between memory item and test item (i.e., change magnitude) is manipulated (Fukuda, Vogel, Mayr, \& Awh, 2010; Scolari, Vogel, \& Awh, 2008). The rationale is that precise memory is necessary to detect a small change from memory items to test items (e.g., red changes into pink), whereas imprecise memory may be sufficient to detect a large change (e.g., red changes into green).

Based on this rationale, precision of visual STM representation can be inferred based on the extent to which mnemonic precision constraints change-detection performance on trials 
with small change magnitudes after STM capacity is factored out using trials with large change magnitudes (Barton, Ester, $\&$ Awh, 2009). Although visual STM quantity and quality can be dissociated using this approach (Fukuda et al., 2010; Scolari et al., 2008), it largely depends on the two levels of change magnitudes. If the large change magnitude is not large enough, the compound measure of performance, $\mathrm{K}$, will be jointly determined by both quantitative and qualitative aspects of memory. If the small change magnitude is not sufficiently small, then the estimate of memory quality based on changedetection performance will be insensitive to subtle changes in mnemonic precision. More problematically, change-detection performance across small and large change magnitudes could be supported by different memory processes or representations (such as fine-grained item representation and coarsegrained configural representations; Xie \& Zhang, 2017a). Finally, the operational definition of precision based on change magnitude manipulation may be uninterpretable considering the underlying decisional process (for details, see Morey, Morey, Brisson, \& Tremblay, 2012).

To address these issues, the present study applied the mixture model to receiver operating characteristic (ROC) data to directly assess the number and quality of visual STM representations. ROC is the function relating cumulative hit rates to cumulative false alarm rates at different levels of response criteria based on the signal detection theory (SDT; Wickens, 2001; also see the Method section). Different aspects of memory representations manifest as different ROC curves. Specifically, the presence or absence of memory (i.e., highthreshold component; HT) yields a linear ROC curve (see Fig. 1a Rouder et al., 2008); whereas a noisy memory signal (i.e., signal-detection-theory component) results in a curvilinear ROC curve (see Fig. 1b; Wilken \& Ma, 2004). Consequently, based on the conceptualization of Zhang and Luck's (2008) mixture model, performance in STM tasks can be modeled as the mixture of the two distinct components: the HT component representing whether the tested item is present in STM or not and the SDT component $\left(d^{\prime}\right)$ for its variable quality/strength when the test item is retained. The overall ROC curve is thus curvilinear and asymmetrical (see the solid line in Fig. 1c). This implementation of Zhang and Luck's mixture model in ROC is analytically similar to a mixture model of recognition memory (DeCarlo, 2002, 2010), in which the proportion of out-of-memory ("guessing") responses were modeled by the proportion of degraded signal that could not be distinguished from noise (see Fig. 1 in DeCarlo, 2002). Note, this model can produce an SDT type of ROC (dashed line in Fig. 1d; e.g., Wilken \& Ma, 2004) or a high-threshold type of ROC (solid line in Fig. 1d; e.g., Rouder et al., 2008). This conceptualization of STM representation (Zhang \& Luck, 2008) can thus be conceptually characterized as a some-or-none model (e.g., Kelley \& Wixted, 2001; Sherman, Atri, Hasselmo, Stern, \& Howard, 2003), in contrast to pure probabilistic high threshold models (Rouder et al., 2008) or pure strength-based SDT models (Wilken \& Ma, 2004).

The two aspects of memory were thus dissociated in the present study using ROC and experimental manipulations. First, to establish the validity of the proposed ROC mixture model, Experiment 1 validated the estimates from the ROC mixture model of change-detection performance against the estimates from the mixture model of recall performance and found strong correlations of the precision estimates between change-detection and continuous-recall tasks, and correlations of the estimates of the number of retained STM representations between the two tasks. Second, Experiments 2 and 3 tested whether the quantity and quality measures from the change-detection task can be experimentally dissociated using selective experimental manipulations. Specifically, different levels of white noise presented simultaneously with the memory items in Experiment 2 reduced the precision of retained visual STM representations without significant effects on the number of retained visual STM representations (Zhang \& Luck, 2008). In contrast, Experiment 3 manipulated the number of encoded stimuli by presenting pattern masks shortly after the onset of the memory array to interrupt STM consolidation of fragile sensory memory into stable STM (Vogel, Woodman, \& Luck, 2006; Zhang \& Luck, 2008). The white-noise masking (Experiment 2) and STM consolidation masking (Experiment 3) produced selective effects on precision and the number of retained STM representations, respectively, for both change-detection and continuous-recall tasks. Altogether, these results have established that the number and precision of visual STM representations in change detection can be modeled using the proposed ROC mixture model and, more importantly, that they can be dissociated psychologically using selective experimental manipulations.

\section{Experiment 1}

Experiment 1 adopted an individual-differences approach to assess the measures of memory quantity and quality and their correlations between recall and change-detection paradigms in visual STM. Participants were presented with a brief memory array of four colors. After a short blank delay interval, either the recall or change-detection task was presented. Note that the two tasks were randomly intermixed so that during the encoding and delay interval the participants could not predict which task they would receive later. Consequently, memory encoding and the resulting memory representations should be similar for the two tasks. This manipulation made it possible to assess whether the ROC mixture model for change detection could produce similar estimates of memory quality and similar estimates of quantity as the recall task when the underlying memory representations were comparable. For the same 

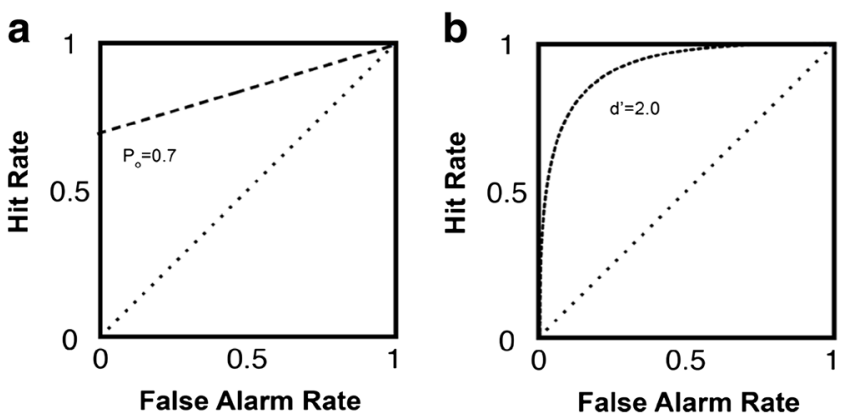

Fig. 1 Theoretical ROCs for Zhang and Luck (2008) mixture model for visual STM in change-detection task with confidence reports. Based on this mixture model, visual STM representations can be decomposed into two distinct aspects: (a) a high threshold (HT) component (linear ROC, $\mathrm{P}_{\mathrm{o}}$ represents the probability that a given stimulus is retained in visual STM) and (b) a SDT component (symmetrical and curvilinear ROC, $d^{\prime}$ represents mnemonic quality/resolution of noisy memory

reason, this experiment was not designed to test whether similar memory processes and representations were shared between recognition and recall in general.

\section{Method}

\section{Participants}

Thirty participants $(18.47 \pm 5.84[M \pm S D]$ years old, 13 males) took part in Experiment 1 for course credits at the University of California, Riverside. All participants had normal color vision and normal (or corrected-to-normal) visual acuity. All participants provided written informed consent prior to the experiment.

\section{Stimuli}

Stimuli were presented on an LCD monitor (calibrated with an $\mathrm{X}$-Rite I1Pro spectrophotometer) with a gray background (6.1 $\mathrm{cd} / \mathrm{m}^{2}$ ) at a viewing distance of $57 \mathrm{~cm}$. The memory array of the change-detection and continuous-recall tasks consisted of four distinct colors, randomly chosen from 180 colors that were evenly distributed on a circle in the Commission Internationale de l'Eclairage (CIE) Lab color space (see Zhang \& Luck, 2008, for details). These colors were at least $24^{\circ}$ from each other in the color space and presented in squares $\left(2^{\circ} \times 2^{\circ}\right)$ at locations randomly selected from six possible locations that were equally spaced on an invisible circle with a radius of $6^{\circ}$. All colors had equal luminance and varied mainly in hue and slightly in saturation. The test array of the continuous-recall task contained four empty outline squares and a color wheel with all 180 colors from the chosen color space. The four outline squares were presented at the original locations and in the same sizes of the original memory items. The color wheel (thickness $=2.2^{\circ}$ ) was presented at the center of the screen, with a radius of $8.2^{\circ}$. The four outline squares
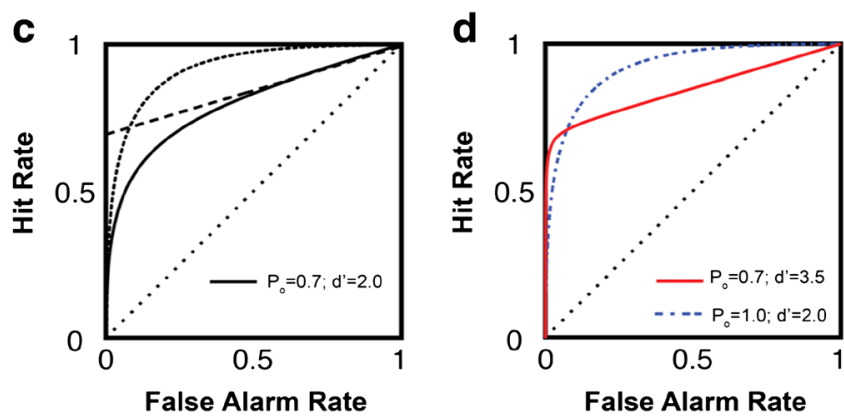

representations). When these two components are mixed together, the resulting ROC (solid line; $\mathbf{c}$ ) is curvilinear and asymmetrical. $\mathbf{d}$ This mixture model can approach HT model as $d^{\prime}$ increases (e.g., increased linearity, solid line) or SDT models as $\mathrm{P}_{\mathrm{o}}$ increases (e.g., increased curvature; dashed line). Consequently, the mixture model can account for pure HT ROCs and SDT ROCs for visual STM. (Color figure online)

were presented at the original locations and in the same sizes of the original memory items. One of these squares had thicker outline than others, indicating the to-be-recalled color. The test array of the change-detection task also contained four squares in the same size as the memory items presented at the original locations. One of the squares contained a color that was either the same as (no-change trials) or different from (change trials) the corresponding memory color (at the same location) from the memory array. Change and no-change trials were equally likely. On change trials, the new color was randomly sampled from the 180 colors, with at least $40^{\circ}$ difference from the corresponding memory color and with at least $24^{\circ}$ difference from other colors in the memory array.

\section{Procedure}

As shown in Fig. 2, each trial started with an 800-ms fixation (not shown in Fig. 2), followed by a 400-ms to-beremembered memory array of four color squares. After a 1,000-ms delay interval, a test array for either the changedetection task or the recall task (in random order, but equally likely across trials) appeared on the screen. In the recall task (see Fig. 2a), the test array contained four empty squares at the locations of original memory items. One of the four squares was highlighted. Participants recalled its color from memory and then matched it to a color on the continuous color wheel, as precisely as possible, using a computer mouse. The mouse cursor always started at the center of the screen at the onset of the test display. Accuracy was stressed over the speed, and responses were not timed. The color participants picked on the color wheel was then marked using a cross. Immediately after, an arrow pointing to correct color value (presented in the memory array) on the wheel was presented for $1,000 \mathrm{~ms}$ as a feedback. The angular deviation between the reported color and the true color indicated the magnitude of error in the recall response. 


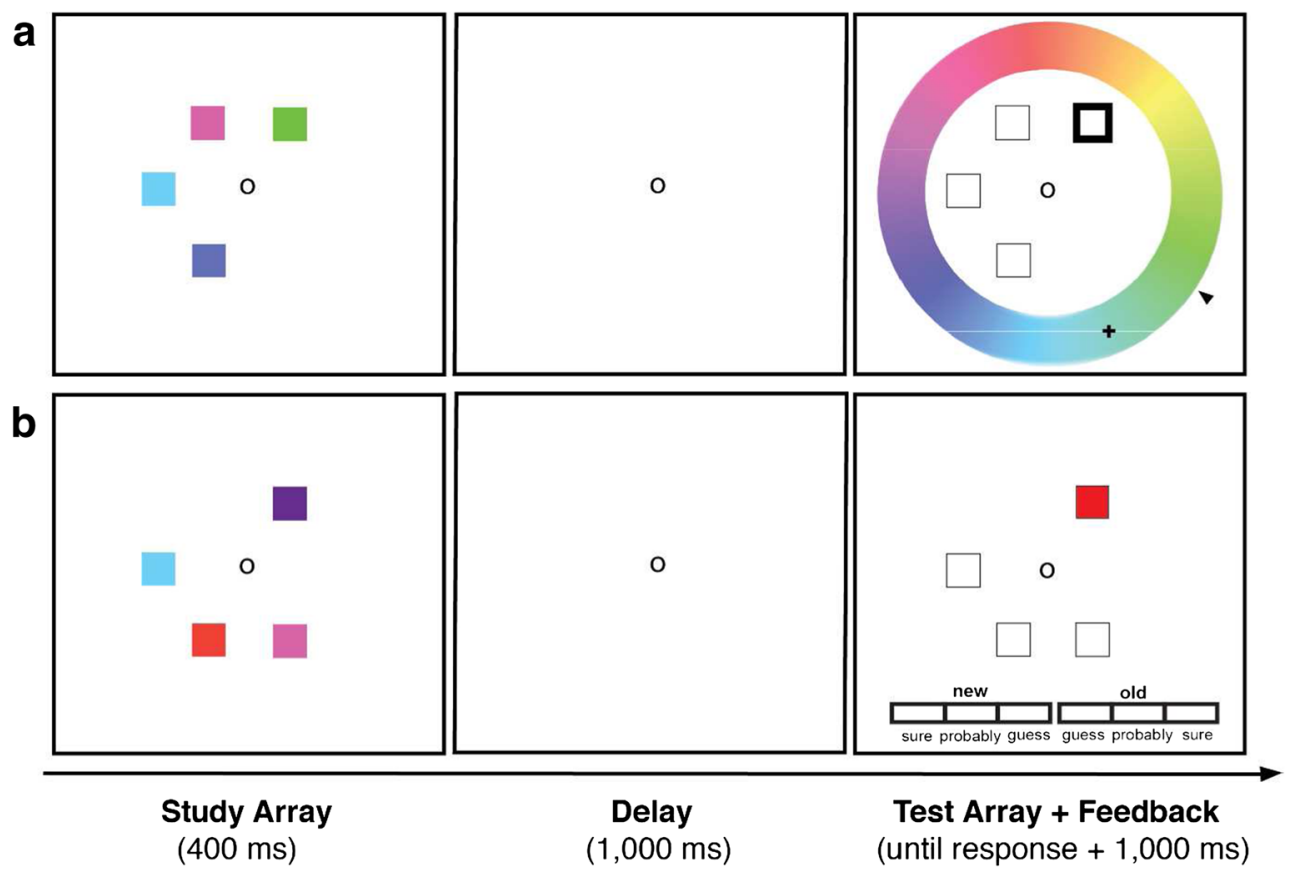

Fig. 2 The continuous color-recall task (a) and the color changedetection task with confidence report (b). After an 800-ms fixation (not shown here), a sample array of four colored squares was presented for 400 ms. After a 1,000-ms delay interval, a test array was presented until responses. It consisted of a continuous color wheel of 180 equally spaced colors and four empty square for the color-recall task (a). Participants tried to reproduce the memory color of a highlighted square by clicking on the best matching color on the color wheel. In the changedetection task (b), the test array consisted of one colored square and three empty squares presented along with a 6-point confidence rating scale. Participants reported whether the tested color was new or old as compared to the color of the corresponding item in the sample array, along with confidence report, from sure to guess. (Color figure online)
In the change-detection task (see Fig. 2b), the test array contained one color and three empty squares at the locations of original memory items. The color presented in the test array was the same as the one at the same location in the memory array (i.e., old) on half of trials, and was a new color that did not appear in the memory array (i.e., new) on the other half of the trials. Participants reported whether the test item was new or old, along with the level of confidence for this response (sure, probably, or guessing) by clicking the mouse cursor on a 6-point confidence scale presented below the test array. A "beep" sound was presented for 1,000 ms after an incorrect old or new response as a feedback. Each participant completed 180 trials for the color-recall task and 180 trials for the changedetection task (90 new and 90 old trials), yielding a total of 360 trials. The color-recall task and the change-detection task were randomly intermixed across trials within blocks of 60 trials (six blocks in total).

\section{Data analysis}

Analysis for recall performance For each trail in the continuous-recall task, recall error was calculated as the angular difference between presented color in the memory array and reported color (ranged from $-180^{\circ}$ to $180^{\circ}$ ). The distributions of the recall errors across trials were then fitted with
Zhang and Luck (2008) mixture model using maximum likelihood estimation (MLE) procedure, separately for each subject at each condition. According to this model, the overall distribution of recall errors is a weighted summation of two components. That is, a von Mises distribution representing graded and noisy memory representations for probed items that are encoded in memory and a uniform distribution for guessing responses when the probed items were not successfully retrieved from memory. The proportion/weight of von Mises distribution represents the probability that the probed item is successfully retrieved $\left(\mathrm{P}_{\mathrm{m}}\right)$. The number of remembered stimuli in visual STM (denoted as $\mathrm{K}_{\text {recall }}$ ) is, therefore, the product of memory set size (the number of to-bemembered items) and $\mathrm{P}_{\mathrm{m}}$. The width of the von Mises distribution, defined as the standard deviation $(S D)$ of the von Mises distribution, is inversely related to memory precision. That is, smaller $S D$ means higher visual STM precision. Overall, this mixture model accounted for more than $98 \%$ variance in the observed data (adjusted $R^{2}$ ).

ROC analysis for change detection performance In the change-detection task, the old or new responses were classified as hits (old responses for no-change trials) or false alarms (old response for change trials). Note that no-change (sameness) was defined as the signal for SDT analyses. 
Across trials, ROC can be constructed from the cumulative hits and false alarms across confidence levels. Specifically, different points on a ROC curve reflect different levels of decisional criteria. The leftmost point represents the hit rate (proportion of sure-old responses for no-change trials) and false alarm rate (proportion of sure-old responses for change trials) at the most conservative decisional criterion. The next point on ROC (moving rightward) represents cumulative hit rate (proportion of sure-old and probably-old responses for no-change trials) and cumulative false alarm rate (proportion of sure-old and probably-old responses for change trials) at a less conservative decisional criterion. This procedure was repeated until cumulative hit rates and false alarm rates were aggregated across all confidence levels (yielding hit rate of one and false alarm rate of one, which is often omitted in data presentation and analysis because it is not informative). The empirical ROCs were then fitted with the ROC mixture model using MLE procedure and the ROC toolbox (Koen, Barrett, Harlow, \& Yonelinas, 2016) for each individual at each condition.

Specifically, cumulative hit rates and false alarm rates from this ROC mixture model can be formally defined as

$$
\begin{aligned}
& \left.\mathrm{P}_{(\text {Hit } \mid \mathrm{x}>=\mathrm{c}}\right)=\mathrm{P}_{\mathrm{o}} \times \Phi\left(c-d^{\prime}\right)+\left(1-\mathrm{P}_{\mathrm{o}}\right) \times \Phi(\mathrm{c}) \\
& \mathrm{P}(\mathrm{FA} \mid \mathrm{x}>=\mathrm{c})
\end{aligned}
$$

Here, $\mathrm{P}_{(\mathrm{Hit} \mid x>=\mathrm{c})}$ and $\mathrm{P}_{(\mathrm{FA} \mid x>=\mathrm{c})}$ represented cumulative hit rates and false alarm rates, respectively, for a response criterion $x$ that was greater or equaled to the confidence level $c . \mathrm{P}_{\mathrm{o}}$ (i.e., the probability of successful retrieval for old items) and $\mathrm{P}_{\mathrm{n}}$ (i.e., the probability of recognizing new items) represent the HT component for old and new items, respectively. This double-threshold component is common for modeling perception and STM data (Aly \& Yonelinas, 2012; Xie \& Zhang, $2017 \mathrm{a}, \mathrm{b})$. The number of retained memory representations in the change-detection task $\left(\mathrm{K}_{\mathrm{CD}}\right)$ is thus the product of $\frac{(P o+P n)}{2}$ and the number of to-be-remembered stimuli (Wickens, 2001). The SDT component $\left(d^{\prime}=\frac{\text { Change Magnitude }}{\text { Aggregated Variance }}\right)$ represent how distinctive a given memory representation is as compared to internal noise. When the change magnitude is predefined (e.g., as an experimental manipulation), $d^{\prime}$ is inversely related to the variability of retained internal representations, allowing $d^{\prime}$ to be used as the index for mnemonic precision of noisy memory representations (similar to DeCarlo, 2002). Note that the two measures $\left(\mathrm{K}_{\mathrm{CD}}\right.$ and $\left.d^{\prime}\right)$ are analytically independent of each other.

In brief, mnemonic precision was defined as $d^{\prime}$ (Wickens, 2001), whereas the number of remembered stimuli was defined as $K_{C D}\left(\frac{\text { Set Size } \times(P o+P n)}{2}\right)$. Overall, this ROC mixture model accounted for more than $98 \%$ variance in the observed data (adjusted $R^{2}$ ). Three alternative models, including the unequal variance signal detection (UVSD) model (see Parks \& Yonelinas, 2007; Wixted, 2007, for details), the dualprocess signal detection model (see Yonelinas \& Parks, 2007 for details), and the two-parameter mixture model (with $\mathrm{P}_{\mathrm{o}}$ and $d^{\prime} ; \mathrm{P}_{\mathrm{n}}$ fixed at zero) were compared to the threeparameter ROC mixture model (with $\mathrm{P}_{\mathrm{o}}, \mathrm{P}_{\mathrm{n}}$, and $d^{\prime}$ ) using a formal model comparison procedure (see Xie \& Zhang, 2017b, and the Online Supplementary Material for details). Given that the three-parameter ROC mixture model provided better overall fits of the observed ROC data than the three alternative models in the present study (see the Online Supplementary Material) and in a previous visual STM study (Xie \& Zhang, 2017b), the three alternative models were not considered in further data analyses.

\section{Results and discussion}

The scatterplots in Fig. 3 clearly demonstrated relationships between the two quantity measures (see Fig. 3a) and between the two precision measures (see Fig. 3d) across the two tasks. First, $\mathrm{K}_{\mathrm{CD}}$, the quantity measure from the ROC mixture model for the change-detection task, and $\mathrm{K}_{\text {recall }}$, the quantity measure from the mixture model for the recall task, were highly correlated with one another $(r=.49[.16, .72], p=.0058$; see Fig. 3a). Second, the precision measure from the ROC model, $d^{\prime}$, was significantly correlated with $S D$, the precision measure from the recall paradigm $(r=-.53[-.75,-.21], p=.0028$; see Fig. $3 \mathrm{~d}$ ). Note, the correlation between $d^{\prime}$ and $S D$ was numerically negative because $S D$ was inversely related to precision, and these correlations remained statistically significant $(p<$ $.05)$, even after Bonferroni correction.

In contrast, there was no significant correlation between quantity measure and precision measure across the two tasks. That is, $\mathrm{K}_{\mathrm{CD}}$ for the change-detection task was not significantly correlated with $S D$ for the recall paradigm $(r=.04[-.32$, $.40], p=.82$; see Fig. $3 \mathrm{~b}$ ). In addition, the precision measure from the ROC model, $d^{\prime}$, was not significantly correlated with $\mathrm{K}_{\text {recall }}(r=.12[-.25, .46], p=.52$; Fig. 3c). Critically, the magnitude of the correlation between $\mathrm{K}_{\mathrm{CD}}$ and $\mathrm{K}_{\text {recall }}$ was significantly greater than that between $\mathrm{K}_{\mathrm{CD}}$ and $S D(z=$ $1.92, p=.027$, one-tailed), based on a one-tailed test on correlated correlations proposed by Meng, Rosenthal, and Rubin (1992). Similarly, the magnitude of the correlation between $d^{\prime}$ and $S D$ were also significantly greater than that of the correlation between $d^{\prime}$ and $\mathrm{K}_{\text {recall }}(z=1.77, p=.038$, one-tailed). In addition, the quantity and quality measures did not significantly correlate with each other within the change-detection task or within the recall task ( $p$ s > .05).

In summary, $\mathrm{K}_{\mathrm{CD}}$ from the change-detection task was significantly correlated with $\mathrm{K}_{\text {recall }}$, but not with $S D$, from the recall task; in contrast, $d^{\prime}$ from the change-detection task was significantly correlated with $S D$, but not with $\mathrm{K}_{\text {recall }}$, from the 

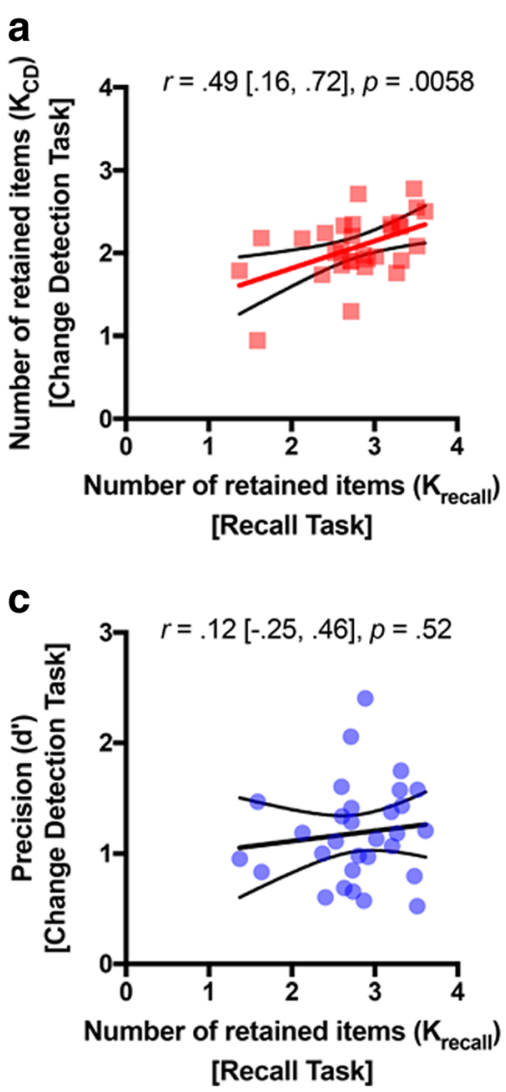

Fig. 3 Correlations of parameters in Experiment 1. a Significant correlation between $\mathrm{K}_{\text {recall }}$ and $\mathrm{K}_{\mathrm{CD}}$; $\mathbf{b}$ nonsignificant correlation between $S D$ and $\mathrm{K}_{\mathrm{CD}}$; $\mathbf{c}$ nonsignificant correlation between $\mathrm{K}_{\text {recall }}$ and $d$ '; d significant correlation between $S D$ and $d$ '. The correlation

recall task. These specificities in the correlations of the parameters across the two tasks could rule out an alternative interpretation that the large correlations between corresponding measures were spurious because better performances on different tasks within participants would inherently correlate with one another. Overall, these results established psychological meanings of two parameters from the ROC mixture model by demonstrating their high consistency (with considerable effect sizes) with the two corresponding measures from the recall paradigm (Brady et al., 2013; Zhang \& Luck, 2008).

\section{Experiment 2}

Experiment 1 provided some preliminary evidence supporting the proposed ROC mixture model in change detection, using the recall task as a benchmark. It is still unclear whether the two measures from change detection could be experimentally dissociated. That is, does experimental manipulations of memory precision only affect the precision measure of the ROC mixture model and does experimental manipulations of the number of retained stimuli only affect the quantity measure of the ROC mixture model? More importantly,
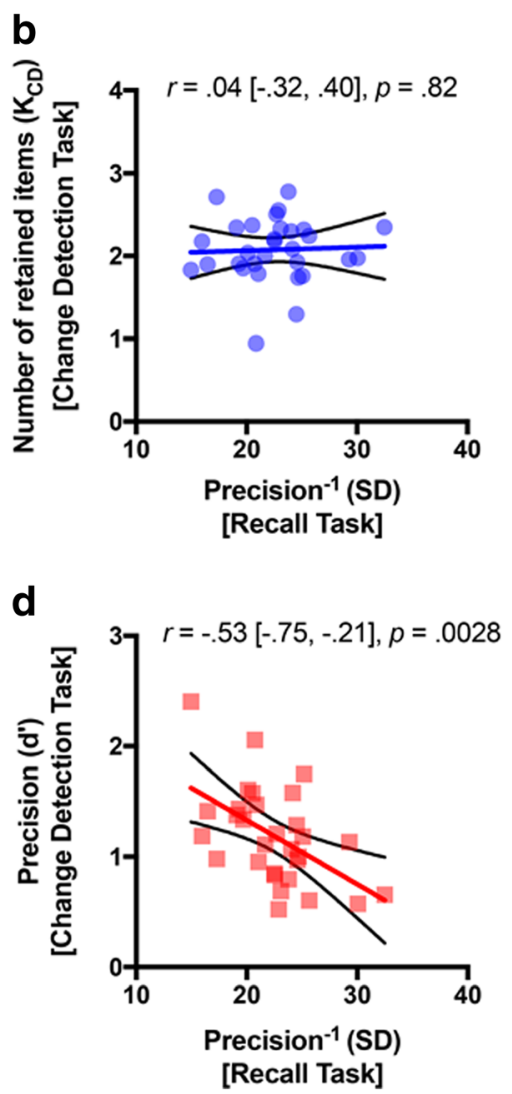

coefficients, along with their $95 \%$ confidence intervals, are presented on top of each panel. Solid and dashed lines represent linear regression fits of the data and their $95 \%$ confidence intervals, respectively. (Color figure online)

considering that recall and change-detection tasks are rarely intermixed in empirical studies, could these dissociations manifest even when memory encoding is optimal for a change-detection task (at least when there is no incentive for participants to recruit similar memory processes for change detection as recall)?

Experiment 2 thus tested whether an experimental manipulation of visual STM precision selectively changed the ROC measure of precision but not the ROC measure of memory quantity. Specifically, the sensory-masking procedure from Experiment 4 in Zhang and Luck (2008) was adopted to manipulate mnemonic precision. In this procedure, white-noise masking (randomly colored dots overlaid on top of the to-be-remembered colors in the memory array; see Fig. 4) was applied to memory items to degrade sensory encoding given that white noise could be integrated into sensory encoding of the signal (integration masking; Enns \& Di Lollo, 2000). The resulting sensory representations with reduced precision were then consolidated into STM (Vogel et al., 2006), leading to less precise STM representations. In the meantime, the sensory degradation was unlikely to reduce the possibility of successful visual STM encoding, unless sensory masking was extremely strong, such that highly imprecise representations would fail to be consolidated into STM (Cappiello 


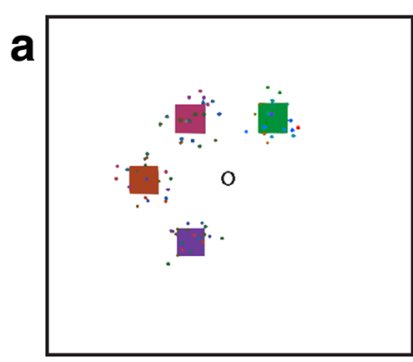

Study Array Low Noise

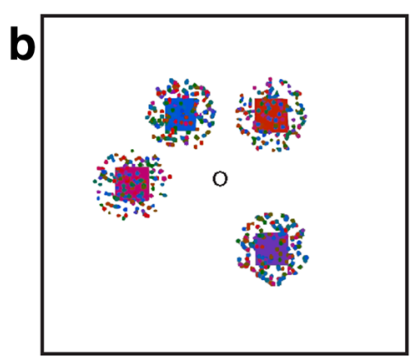

Study Array

High Noise
Fig. 4 Examples of the low noise (a) and high noise $(b)$ conditions for white-noise masking of the memory array in Experiment 2. (Color figure online)

\& Zhang, 2016). As an extreme example, heavy noise masking could entirely overwrite the to-be-remembered color squares, leading to little information perceived for the memory items. Experiment 2 thus used similar levels of noise masking (numbers of randomly colored dots; see Fig. 4) as those in Zhang and Luck (2008), which previously produced a selective reduction of mnemonic precision in the continuous-recall task (Zhang \& Luck, 2008). The same levels of white-noise masking were used across the change-detection and color-recall tasks.

To ensure optimal memory encoding and retrieval for either recognition for the change-detection task or recall for the continuous color-recall task, change-detection and recall tasks were blocked with the order counterbalanced across participants. We predicted less precise STM representations (larger $S D$ for the color-recall task and smaller $d^{\prime}$ for the change-detection task) at high-noise condition relative to low-noise condition. In contrast, the number of retained STM representations $\left(\mathrm{K}_{\text {recall }}\right.$ for the color-recall task and $\mathrm{K}_{\mathrm{CD}}$ for the change-detection task) should be similar between the two noise conditions.

\section{Method}

\section{Participants}

Twenty new participants ( $20.56 \pm 4.72$ years, nine males) took part in Experiment 2 for course credit at the University of California, Riverside. All participants reported having normal color vision and had normal (or corrected-to-normal) visual acuity. All participants gave informed consent at the beginning of the experiment.

\section{Stimuli and procedure}

The stimuli and procedure were the same as Experiment 1, with these following exceptions. First, white-noise masking was added to the memory array. The white-noise patterns consisted of 20 (low-noise condition; see Fig. 4a) or 150 (high-noise condition; Fig. 4 b) randomly colored dots $\left(0.17^{\circ}\right.$ $\times 0.17^{\circ}$ in size) that were distributed pseudorandomly within a circle of radius $3^{\circ}$ with homogeneous density across eccentricity and angular degrees. The colors of these dots were randomly sampled from the 180 colors on the color wheel. The magnitude of white-noise masking, in terms of the numbers of random colored dots, and the duration of memory array were chosen to produce a large change in visual STM precision based on pilot testing and a previous study (Zhang \& Luck, 2008). Second, for the change-detection task, a larger change magnitude from the memory item to the changed item in the test array (at least $50^{\circ}$ difference in the color space, as opposed to $40^{\circ}$ in Experiment 1) was used to avoid potential floor performance due to noise masking. Third, the recall and change-detection tasks were blocked, but the two experimental conditions (high noise vs. low noise) were randomly intermixed within experimental blocks for each task. Each participant completed 360 trials across three blocks for the color-recall task and 360 trials across three blocks for the change-detection task (180 new and 180 old trials), yielding a total of 720 trials. The order of the recall task and changedetection task was counterbalanced across participants.

\section{Results and discussion}

Replicating previous findings for the recall paradigm (Zhang \& Luck, 2008), the white-noise integration masking manipulation significantly reduced mnemonic precision (manifested as a wider peak in the overall error distribution in Fig. 5a) from the low-noise condition to the high-noise condition in the color-recall task, $t(19)=3.32, p=.0036$, Cohen's $d=0.74$, Bayes factor $=12.13$; see Fig. $5 \mathrm{c}$. In contrast, the white noise did not significantly change $\mathrm{K}_{\text {recall }}$, the number of encoded STM representation in the recall task, $t(19)=0.88, p=.39$, Bayes factor $=0.33$; see Fig. $5 b$.

More importantly, this selective reduction of mnemonic precision by the white noise also manifested in the changedetection task. There was a significant reduction in mnemonic precision (manifested as less curved ROC in Fig. 5d) from the low-noise condition to the high-noise condition, $t(19)=3.53$, $p=.0022$, Cohen's $d=0.79$, Bayes factor $=18.22$; see Fig. 5 f. In contrast, the difference in $\mathrm{K}_{\mathrm{CD}}$ between the low-noise and high-noise conditions was not significant, $t(19)=0.78, p=$ .44 , Bayes factor $=0.30$; see Fig. 5 e. In addition, the experimental effects of white-noise masking seemed to be consistent between the two paradigms across participants. Specifically, the experimental effect on $S D$ in the recall task (i.e., $\left[\left(\mathrm{SD}_{\text {HighNoise }}-\mathrm{SD}_{\text {LowNoise }}\right) / \mathrm{SD}_{\text {Average }}\right]$; Xie \& Zhang, 2017c) was significantly correlated $(r=-.50, p=.023)$ with that on $d^{\prime}$ in the recognition task $\left[\left(d_{\text {HighNoise }}^{\prime}-d_{\text {LowNoise }}^{\prime}\right) / d_{\text {Average }}^{\prime}\right]$ across participants. Note, the numerical value of this correlation is negative because $S D$ is inversely related to precision. Overall, these results indicated that the precision measure, $d^{\prime}$, from the ROC mixture model for the change-detection task 

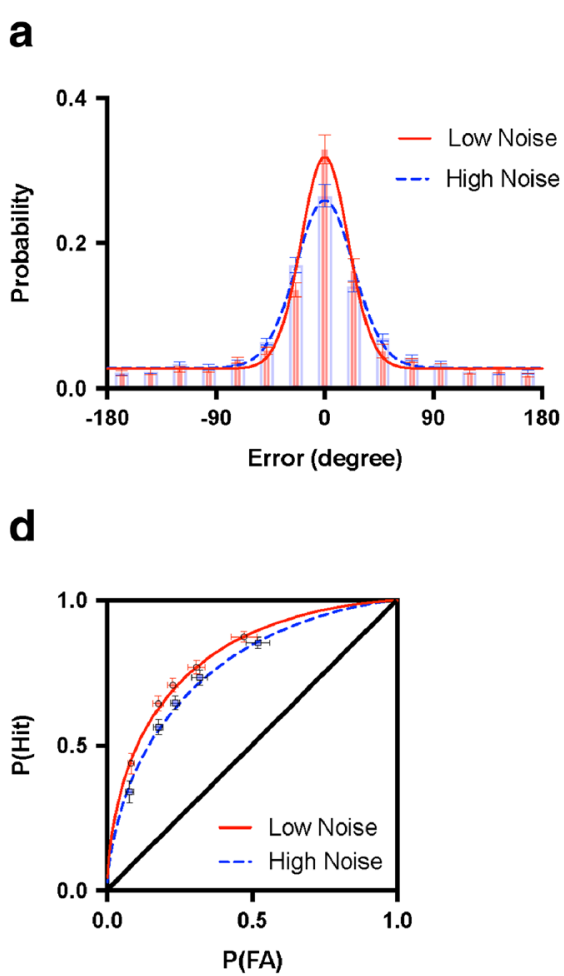

Fig. 5 Results for the recall task (a-c) and the change-detection task (df) from Experiment 2. a Aggregated recall error distributions across the two experimental conditions. High noise masking produced a wider recall error distribution (hence, reduced precision for visual STM). b $\mathrm{K}_{\text {recall }}$ and c $S D$ for the two experimental conditions of the recall task. d Aggregated

sufficiently tracked the changes in mnemonic precision due to white-noise masking of the memory items.

\section{Experiment 3}

Experiment 3 tested whether $\mathrm{K}_{\mathrm{CD}}$ from the change-detection task was sensitive to the changes in the number of encoded STM representations. Consolidation-masking procedure (Vogel et al., 2006; Zhang \& Luck, 2008), a type of interruption masking (Enns \& Di Lollo, 2000), was used to introduce changes in the number of encoded STM representations over the period of time allowed for STM consolidation. Specifically, pattern masks were presented after the memory array with a stimulus onset asymmetry (SOA) of $117 \mathrm{~ms}$ (short SOA condition) or $340 \mathrm{~ms}$ (long SOA condition) to interrupt consolidation of fragile sensory representations into visual STM (see Fig. 6). Memory-array duration was also reduced to $100 \mathrm{~ms}$ so that consolidation masking could be effective. Similar to Experiment 2, the two tasks were blocked in Experiment 3, but the masking conditions were randomly mixed within experimental blocks for each task.

It was previously demonstrated that this consolidationmasking manipulation could lead to a substantial increase in
Recall Task

n.s.

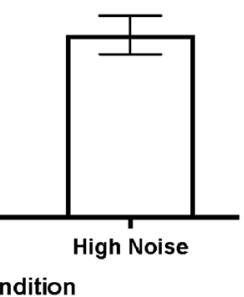

Condition

C

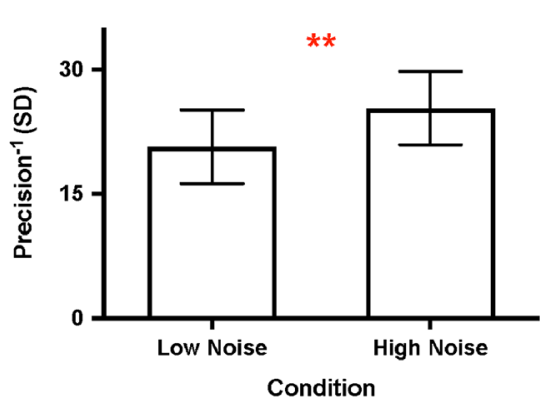

f

Change Detection Task

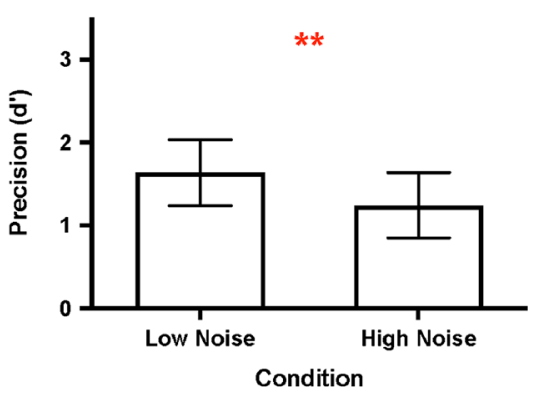

ROCs across the two experimental conditions. e $\mathrm{K}_{\mathrm{CD}}$ and $\mathbf{f} d^{\prime}$ for the two experimental conditions of the change-detection task. Error bars in a and d represent standard error. Error bars in $\mathbf{b}-\mathbf{c}$ and $\mathbf{e}-\mathbf{f}$ represent withinsubject $95 \%$ confidence intervals (Morey, 2008). **. $p<.01$. n.s. $=$ not significant. (Color figure online)

the number of encoded stimuli, without significant effect on the precision of successfully encoded visual STM representations, as the memory-to-mask SOA increased in the recall task (Zhang \& Luck, 2008). It was thus predicted that more representations (larger $\mathrm{K}_{\text {recall }}$ for the color-recall task and $\mathrm{K}_{\mathrm{CD}}$ for the change-detection task) could be encoded at the long SOA (340 ms) condition as compared to the short SOA (117 ms) condition. In contrast, mnemonic precision ( $S D$ for the colorrecall task and $d^{\prime}$ for the change-detection task) should be comparable across the two SOA conditions.

\section{Method}

\section{Participants}

Twenty new participants $(20.68 \pm 1.33$ years, 11 males $)$ took part in Experiment 3 for course credit at the University of California, Riverside. One additional participant used only four options on the 6-point confidence scale, resulting in insufficient data points for ROC modeling, and was thus excluded from further data analyses (Yonelinas \& Parks, 2007). All participants had normal (or corrected-to-normal) visual acuity and reported having normal color vision. They gave written informed consent at the beginning of the experiment. 


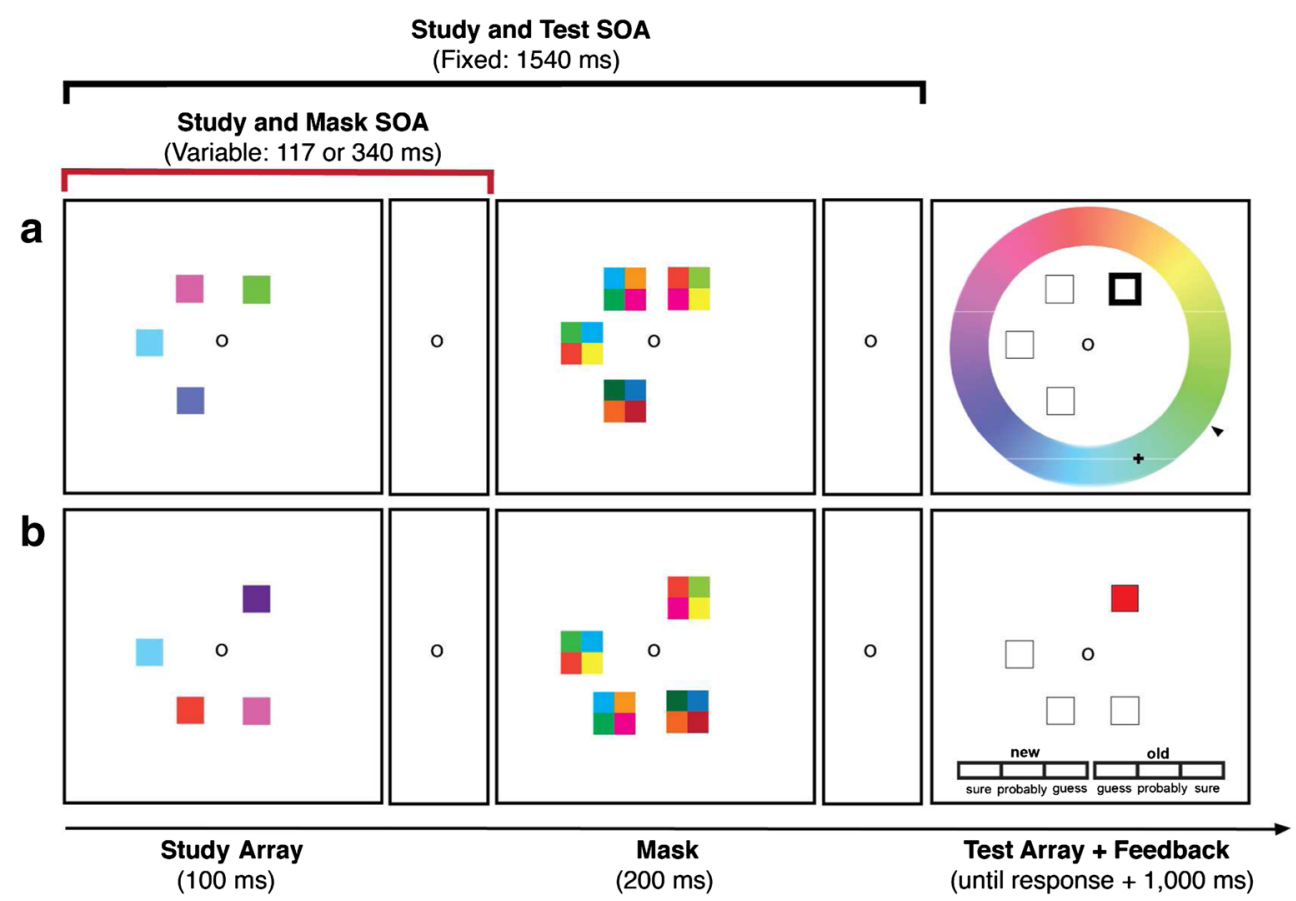

Fig. 6 The consolidation masking procedure in Experiment 3 for the continuous color-recall task (a) and the change-detection task with confidence report (b). In both tasks, a sample array of four colored squares was presented for $100 \mathrm{~ms}$. Pattern masks consisting of four random colors for each colored square were presented for $200 \mathrm{~ms}$ with either a short sample-and-mask stimulus onset asymmetry (SOA) of $117 \mathrm{~ms}$ or a longer sample-and-mask SOA of $340 \mathrm{~ms}$. The test array appeared at a fixed sample-and-test SOA of 1,540 ms. (Color figure online)

\section{Stimulus and procedure}

The stimuli and procedure of Experiment 3 (see Fig. 6) were highly comparable with those of Experiment 2, with the exceptions on the masking stimuli and procedure as follows. First, as shown in Fig. 6, the consolidation-masking stimuli consisted of four masking patterns at the original locations of the memory items. Each masking pattern $\left(2.3^{\circ} \times 2.3^{\circ}\right)$ consisted of four colored squares $\left(1.15^{\circ} \times 1.15^{\circ}\right)$ in a $2 \times 2$ arrangement. The colors of these squares were randomly selected from the 180 colors, without repetition, and completely uncorrelated with the to-be-remembered colors. Second, these pattern masks were presented after the memory array, with the SOA between the memory and mask arrays at $117 \mathrm{~ms}$ (short SOA condition) or $340 \mathrm{~ms}$ (long SOA condition). To allow the short SOA, the duration of the memory array was reduced to $100 \mathrm{~ms}$. Third, the test array appeared with a fixed memoryand-test SOA at 1,540 ms for both short and long memory-tomask SOA conditions.

\section{Results and discussion}

Replicating findings from a previous study (Zhang \& Luck, 2008), the consolidation-masking procedure in the colorrecall task markedly reduced the number of the item encoded into visual STM in the short SOA condition, manifested as a heightened tail in the recall error distribution in the short SOA condition, as compared to the long SOA condition (see Fig. 7a). In contrast, the widths of the recall error distributions were comparable across the SOA conditions. These observations were supported by a significant difference in $\mathrm{K}_{\text {recall }}, t(19)$ $=9.03, p<.001$, Cohen's $d=2.02$, Bayes factor $>5,0000$; see Fig. $7 \mathrm{~b}$, but not in $S D, t(19)=0.65, p=.53$, Bayes factor $=$ 0.28 ; see Fig. $7 \mathrm{c}$, between the SOA conditions.

The results from the change-detection task mirrored the results from the continuous color-recall task. There was a significant difference in the number of encoded items between the short SOA and long SOA conditions, $t(19)=3.16, p=$ .0052 , Cohen's $d=0.71$, Bayes factor $=8.94$; see Fig. 7e, based on $\mathrm{K}_{\mathrm{CD}}$ from the ROC mixture model (see Fig. 7d). In contrast, there was no significant difference in $d^{\prime}$ between the short SOA and long SOA conditions, $t(19)=0.02, p=.98$, Bayes factor $=0.23$; see Fig. 7 f. Overall, these results were in line with the prediction that the consolidation-masking procedure selectively affected the number of stimuli consolidated into STM, with little effect on mnemonic precision of already encoded representations (Zhang \& Luck, 2008). Nevertheless, we failed to observe a significant correlation between the relative change of the number of encoded items $\left[\left(\mathrm{K}_{\mathrm{LongSOA}}-\right.\right.$ $\left.\left.\mathrm{K}_{\text {ShortSOA }}\right) / \mathrm{K}_{\text {Average }}\right]$ in the recall task $\left(\mathrm{K}_{\text {recall }}\right)$ and that in the recognition task $\left(\mathrm{K}_{\mathrm{CD} ;} p>.05\right)$, probably due to measurement noise with a small sample size of Experiment 3. 

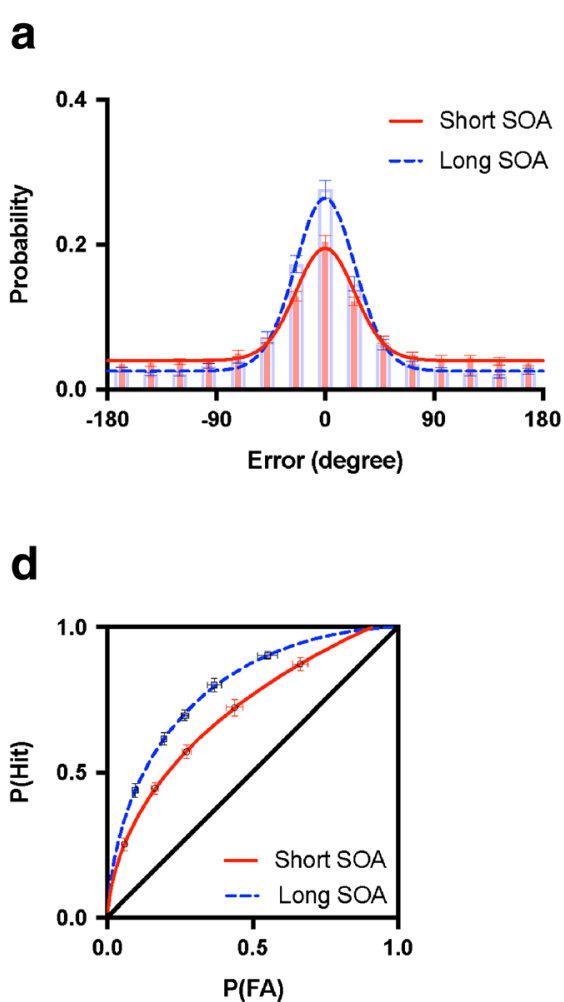

Fig. 7 Results for the recall task (a-c) and the change-detection task (df) from Experiment 3. a Aggregated recall error distributions across the two experimental conditions. The short SOA condition resulted in heightened tails of the recall error distribution (hence, reduced probability of successful memory retrieval), as compared to the long SOA condition. b $\mathrm{K}_{\text {recall }}$ and $\mathbf{c} S D$ for the two experimental conditions

\section{General discussion}

The present study proposed and examined a descriptive model for the qualitative and quantitative aspects of visual STM recognition based on ROC data from change-detection tasks. The quantitative aspect of recognition was modeled as $\mathrm{K}_{\mathrm{CD}}$ representing the number of retained representations in STM. The qualitative aspect of recognition was modeled as an SDT component $\left(d^{\prime}\right)$ representing precision of retained representations. Overall ROCs were modeled as the mixture of the two components, reflecting the some-or-none nature of visual STM representations (Awh, Barton, \& Vogel, 2007; Christopher Donkin, Nosofsky, Gold, \& Shiffrin, 2013; Pratte, Park, Rademaker, \& Tong, 2017; Rouder et al., 2008; Zhang \& Luck, 2008). This ROC mixture model was fitted to empirical ROC data from a change-detection task in Experiment 1, yielding independent measures of the number and precision of retained memory representations. The two measures were strongly and significantly correlated with the corresponding measures from the Zhang and Luck (2008) mixture model for continuous-recall data across participants. These results suggested that the ROC mixture model applied to the change-detection data measured fundamentally
Recall Task

$* *$

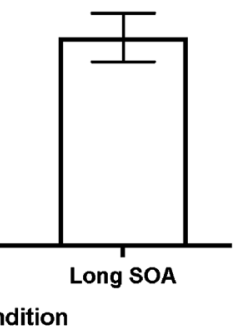

Condition

f

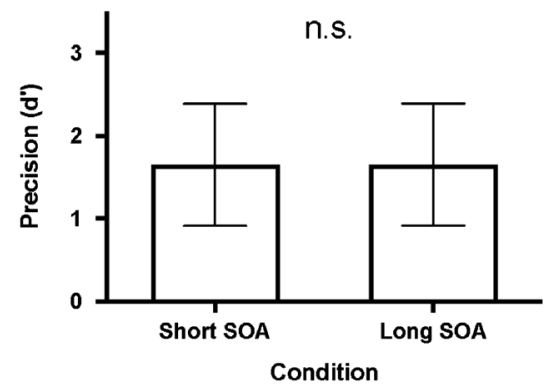

of the recall task. d Aggregated ROCs across the two experimental conditions. e $\mathrm{K}_{\mathrm{CD}}$ and $\mathbf{f} d^{\prime}$ for the two experimental conditions of the change-detection task. Error bars in a and d represent standard error. Error bars in b-c and e-f represent within-subject $95 \%$ confidence intervals. ${ }^{* *} . p<.01$. n.s. $=$ not significant. (Color figure online)

similar aspects of STM as the recall task. Note, the consistency in the corresponding measures between recognition and recall performance may depend on shared memory encoding because these two tasks were randomly intermixed across trials. With this manipulation, participants would not be able to predict how their memory would be tested at the onset of the memory array, and thus this would push participants to rely on the same memory representations to perform both tasks. Experiment 2 and 3 further experimentally dissociated the two measures of change-detection performance by demonstrating the selective effects of the sensory-making procedure on mnemonic precision (Experiment 2) and the selective effects of the consolidation-masking procedure on the number of retained representations (Experiment 3). These dissociable effects mirrored previous findings for the recall task (Zhang \& Luck, 2008), even when the recall and recognition tasks were blocked to maximize memory encoding that was optimal for recall and recognition separately. Together, these results demonstrated that measures of the number and precision of STM representations in change-detection tasks could robustly track individual differences in and experimental effects on the two aspects of visual STM. 


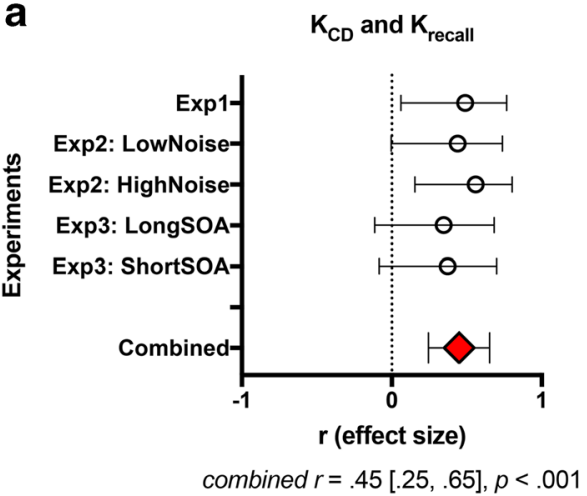

C

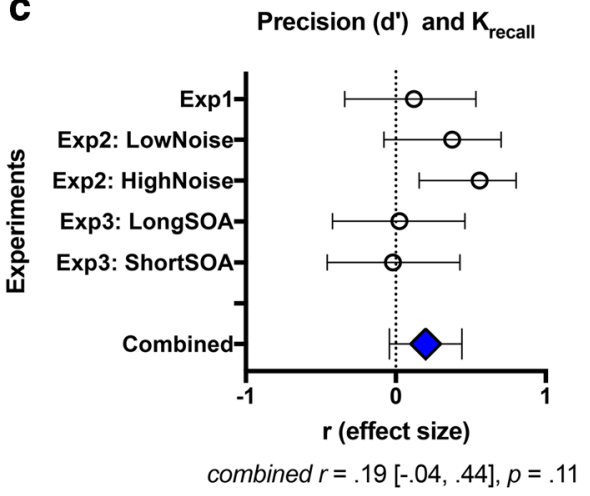

b

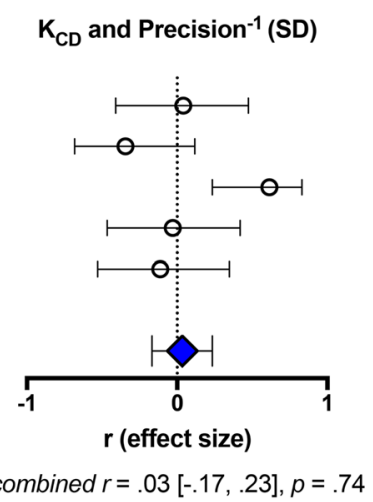

d

Precision (d') and Precision ${ }^{-1}(\mathrm{SD})$

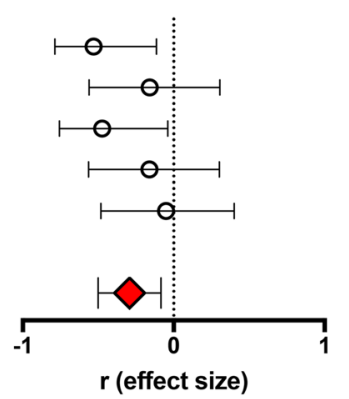

combined $r=-.29[-.08, .50], p=.0057$
Fig. 8 Combining the correlations of model parameters metaanalytically across experiments using a random-effect model after correcting for the nonindependence issue for correlations in Experiment 2 and 3 (see Cheung \& Chan, 2013, for details). Circles represent individual correlations, and diamonds represent combined effect sizes. Whiskers represent the random-effect $95 \%$ confidence intervals. Note. The $95 \%$ confidence intervals for correlations can be asymmetrical because of the bounded nature of $r$. (Color figure online)
Empirically, this study has developed a quantitative method to reliably estimate the probability of remembering and mnemonic precision of remembered information that can be applied to much broader contexts than the original mixture model of visual STM for recall (Zhang \& Luck, 2008). First, we have generalized the distinctions between the quality and quantity of visual STM and their corresponding estimates from recall to recognition. Second, the ROC mixture model has made it possible to estimate the two aspects of internal representations without the previous limits on the physical stimuli (e.g., reproducible features in circular space) and on the assumptions (e.g., how guessing responses are distributed) for the original mixture model of recall (Brady et al., 2013; Zhang \& Luck, 2008). As a result, the two measures of visual STM can be obtained using the proposed ROC mixture model for complex visual stimuli that could not be easily reproduced or quantified (Xie \& Zhang, 2017b).

More importantly, the present model and findings are informative for the current theoretical debate on the nature of the storage limit in visual STM. On the one hand, capacity-limited hypotheses (Cowan, 2001; Luck \& Vogel, 1997; Miller, 1956; Zhang \& Luck, 2008) attribute decreasing performance with the number of to-be-remembered stimuli to capacity (i.e., a limited number of representations can be simultaneously maintained in STM). On the other hand, precision-limited hypotheses attribute the bottleneck of STM storage entirely to changes in mnemonic precision for recall (Bays \& Husain, 2008; Ma, Husain, \& Bays, 2014) and recognition (Devkar, Wright, \& Ma, 2015; Elmore \& Wright, 2015; Elmore et al., 2011; Keshvari, van den Berg, \& Ma, 2013). A recent variant of precision-limited hypotheses, the variable precision model (van den Berg et al., 2012), replaces the capacity limit with variable mnemonic precision in that the variability in precision could produce extremely low precision that behaviorally resembles random memory responses. To test between the two classes of hypotheses, recent research seems to show an increasing emphasis on goodness of fits in cognitive modeling of visual STM (van den Berg, Awh, \& Ma, 2014), even though striving for "ideal" model fits has never been the ultimate goal in cognitive science (see Pitt \& Myung, 2002; Roberts \& Pashler, 2000). Specifically, although a good fit is the starting point of theory development (see the Online Supplementary Material for formal model comparison and model recovery simulation of the current 
data), it should not be an end point (Pitt \& Myung, 2002; Roberts \& Pashler, 2002; Rodgers \& Rowe, 2002a, b). As often observed in the cognitive modeling literature, competing models may fit the data adequately well (Yonelinas \& Parks, 2007), but they provide fundamentally different conceptualizations of the underlying representations and processes (e.g., UVSD vs. DPSD model of recognition memory; Wixted, 2007; Yonelinas \& Parks, 2007). It is thus pivotal to establish the psychological meaning and validity of model parameters, making it possible to generate predictions, which is one of the fundamental goals of theory development (Roberts \& Pashler, 2000).

With evidence from the individual differences approach and the experimental dissociation approach, the current study has validated the psychological meanings of the mixturemodel parameters in both recall and recognition. More importantly, the present study have established the dissociable nature of visual STM quality and quantity using experimental dissociation, indicating that the two aspects of STM representations are theoretically and experimentally independent of each other (Luck \& Vogel, 2013; Xie \& Zhang, 2016; Ye, Zhang, Liu, Li, \& Liu, 2014; Zhang \& Luck, 2008). These findings have therefore provided strong support for capacitylimited hypotheses.

Given that the recall and recognition tasks are typically tested separately in the literature, Experiments 2 and 3 have blocked the two tasks (instead of randomly mixing them within experimental blocks as in Experiment 1). This manipulation is to ensure that the relationships in corresponding aspects of memory across recall and recognition can be generalized to conditions where optimal encoding and retrieval for each task are allowed. Although the experimental manipulations have dissociated the quantitative and qualitative aspects of memory in similar ways across recall and recognition, the increased task-specific variance in Experiment 2 and 3 would make it difficult to obtain significant correlations in corresponding model parameters across recall and recognition. As summarized in Fig. 8, in a mini meta-analysis that combines correlations of corresponding model parameters across experiments after correcting for nonindependence (i.e., different conditions of the same experiment) for correlations in Experiment 2 and 3 (see Cheung \& Chan, 2013, for details), the patterns previously observed in Experiment 1 seem to be robust even after considering evidence with increased "noise" from different task procedures and sample sizes. That is, the quantity estimates from the recall and recognition paradigms are selectively correlated with one another, and the quality estimates are selectively correlated with one another across recall and recognition.

In summary, the present study has developed a ROC mixture model for visual STM. The potential applications of the mixture model in a broader context could extend the assessment of memory quantity and quality into neurobiological research in animals (e.g., Fortin, Wright, \& Eichenbaum, 2004) and into translational and applied research to reveal potentially dissociable neural mechanisms for and functional significances of the two aspects of memory (Koriat \& Goldsmith, 1996; Koriat et al., 2000).

Acknowledgements We would like to thank the constructive suggestions from Chad Dubé and two anonymous reviewers.

Author contributions Both authors designed the study. W.X. collected data and performed data analyses. Both authors contributed to manuscript preparation.

Compliance with ethical standards This study is approved by the Institutional Review Board (IRB) at University of California, Riverside.

Declaration of conflicting interests The authors declared that they had no conflicts of interest with respect to their authorship or the publication of this article.

\section{References}

Alvarez, G. A., \& Cavanagh, P. (2004). The capacity of visual short-term memory is set both by visual information load and by number of objects. Psychological Science, 15(2), 106-111. doi:10.2307/ 40063936

Aly, M., \& Yonelinas, A. P. (2012). Bridging consciousness and cognition in memory and perception: Evidence for both state and strength processes. PLoS ONE, 7(1), e30231. doi:10.1371/journal.pone. 0030231

Awh, E., Barton, B., \& Vogel, E. K. (2007). Visual working memory represents a fixed number of items regardless of complexity. Psychological Science, 18(7), 622-628. doi:10.1111/j.1467-9280. 2007.01949.x

Bae, G. Y., Olkkonen, M., Allred, S. R., Wilson, C., \& Flombaum, J. I. (2014). Stimulus-specific variability in color working memory with delayed estimation. Journal of Vision, 14(4), 7. doi:10.1167/14.4.7

Barton, B., Ester, E. F., \& Awh, E. (2009). Discrete resource allocation in visual working memory. Journal of Experimental Psychology: Human Perception and Performance, 35(5), 1359-1367. doi:10. 1037/a0015792

Bays, P. M., \& Husain, M. (2008). Dynamic shifts of limited working memory resources in human vision. Science, 321(5890), 851-854. doi: $10.1126 /$ science. 1158023

Bernbach, H. A., \& Kupchak, P. G. (1972). Recognition and recall in short-term memory. Journal of Mathematical Psychology, 9(2), 237-242. doi:10.1016/0022-2496(72)90029-6

Brady, T. F., Konkle, T., Gill, J., Oliva, A., \& Alvarez, G. A. (2013). Visual long-term memory has the same limit on fidelity as visual working memory. Psychological Science, 24(6), 981-990. doi:10. $1177 / 0956797612465439$

Cappiello, M., \& Zhang, W. (2016). A dual-trace model for visual sensory memory. Journal of Experimental Psychology: Human Perception and Performance, 42(11), 1903-1922. doi:10.1037/xhp0000274

Cheung, S. F., \& Chan, D. K. S. (2013). Meta-analyzing dependent correlations: An SPSS macro and an R script. Behavior Research Methods, 46(2), 331-345. doi:10.3758/s13428-013-0386-2

Cornelissen, F. W., \& Greenlee, M. W. (2000). Visual memory for random block patterns defined by luminance and color contrast. Vision Research, 40(3), 287-299. 
Cowan, N. (2001). The magical number 4 in short-term memory: A reconsideration of mental storage capacity. Behavioral and Brain Sciences, 24(1), 87-185. doi:10.1177/0963721409359277

DeCarlo, L. T. (2002). Signal detection theory with finite mixture distributions: Theoretical developments with applications to recognition memory. Psychological Review, 109(4), 710-721. doi:10.1037// 0033-295X.109.4.710

DeCarlo, L. T. (2010). On the statistical and theoretical basis of signal detection theory and extensions: Unequal variance, random coefficient, and mixture models. Journal of Mathematical Psychology, 54(3), 304-313. doi:10.1016/j.jmp.2010.01.001

Devkar, D. T., Wright, A. A., \& Ma, W. J. (2015). The same type of visual working memory limitations in humans and monkeys. Journal of Vision, 15(16), 13. doi:10.1167/15.16.13

Donkin, C., Nosofsky, R., Gold, J., \& Shiffrin, R. (2015). Verbal labeling, gradual decay, and sudden death in visual short-term memory. Psychonomic Bulletin \& Review, 22(1), 170-178. doi:10.3758/ s13423-014-0675-5

Donkin, C., Nosofsky, R. M., Gold, J. M., \& Shiffrin, R. M. (2013). Discrete-slots models of visual working-memory response times. Psychological Review, 120(4), 873-902. doi:10.1037/a0034247

Elmore, L. C., Ma, W. J., Magnotti, J. F., Leising, K. J., Passaro, A. D., Katz, J. S., \& Wright, A. A. (2011). Visual short-term memory compared in rhesus monkeys and humans. Current Biology, 21(11), 975-979. doi:10.1016/j.cub.2011.04.031

Elmore, L. C., \& Wright, A. A. (2015). Monkey visual short-term memory directly compared to humans. Journal of Experimental Psychology: Animal Learning and Cognition, 41(1), 32-38. doi: 10.1037/xan0000050

Enns, J. T., \& Di Lollo, V. (2000). What's new in visual masking? Trends in Cognitive Sciences, 4(9), 345-352. doi:10.1016/S1364-6613(00) 01520-5

Fortin, N. J., Wright, S. P., \& Eichenbaum, H. (2004). Recollection-like memory retrieval in rats is dependent on the hippocampus. Nature, 431(7005), 188-191. doi:10.1038/nature02853

Fukuda, K., Vogel, E., Mayr, U., \& Awh, E. (2010). Quantity, not quality: The relationship between fluid intelligence and working memory capacity. Psychonomic Bulletin \& Review, 17(5), 673-679. doi:10. 3758/17.5.673

Gold, J. M., Hahn, B., Zhang, W., Robinson, B. M., Kappenman, E. S., Beck, V. M., \& Luck, S. J. (2010). Reduced capacity but spared precision and maintenance of working memory representations in schizophrenia. Archives of General Psychiatry, 67(6), 570-577. doi: 10.1001/archgenpsychiatry.2010.65

Haist, F., Shimamura, A. P., \& Squire, L. R. (1992). On the relationship between recall and recognition memory. Journal of Experimental Psychology: Learning, Memory, and Cognition, 18(4), 691-702. doi:10.1037//0278-7393.18.4.691

Hardman, K. O., Vergauwe, E., \& Ricker, T. J. (2017). Categorical working memory representations are used in delayed estimation of continuous colors. Journal of Experimental Psychology: Human Perception and Performance, 43(1), 30-54. doi:10.1037/ xhp0000290

Hollingworth, H. L. (1913). Characteristic differences between recall and recognition. The American Journal of Psychology, 24(4), 532. doi: $10.2307 / 1413450$

Huttenlocher, J., Hedges, L. V., \& Duncan, S. (1991). Categories and particulars: Prototype effects in estimating spatial location. Psychological Review, 98(3), 352-376.

Kelley, R., \& Wixted, J. T. (2001). On the nature of associative information in recognition memory. Journal of Experimental Psychology: Learning, Memory, and Cognition, 27(3), 701-722. doi:10.1037/ 0278-7393.27.3.701

Keshvari, S., van den Berg, R., \& Ma, W. J. (2013). No evidence for an item limit in change detection. PLoS Computational Biology, 9(2), e1002927. doi:10.1371/journal.pcbi.1002927
Koen, J. D., Barrett, F. S., Harlow, I. M., \& Yonelinas, A. P. (2016). The ROC Toolbox: A toolbox for analyzing receiver-operating characteristics derived from confidence ratings. Behavior Research Methods, 1-8. doi:10.3758/s13428-016-0796-Z

Koriat, A., \& Goldsmith, M. (1996). Memory metaphors and the real-life/ laboratory controversy: Correspondence versus storehouse conceptions of memory. Behavioral and Brain Sciences, 19(02), 167-188. doi:10.1017/S0140525X00042114

Koriat, A., Goldsmith, M., \& Pansky, A. (2000). Toward a psychology of memory accuracy. Annual Review of Psychology, 51(1), 481-537. doi:10.1146/annurev.psych.51.1.481

Lee, B., \& Harris, J. (1996). Contrast transfer characteristics of visual short-term memory. Vision Research, 36(14), 2159-2166.

Luck, S. J., \& Vogel, E. K. (1997). The capacity of visual working memory for features and conjunctions. Nature, 390(6657), 279-281. doi: $10.1038 / 36846$

Luck, S. J., \& Vogel, E. K. (2013). Visual working memory capacity: From psychophysics and neurobiology to individual differences. Trends in Cognitive Sciences, 17(8), 391-400. doi:10.1016/j.tics. 2013.06.006

Ma, W. J., Husain, M., \& Bays, P. M. (2014). Changing concepts of working memory. Nature Neuroscience, 17(3), 347-356. doi:10. 1038/nn.3655

Makovski, T., Watson, L. M., Koutstaal, W., \& Jiang, Y. V. (2010). Method matters: Systematic effects of testing procedure on visual working memory sensitivity. Journal of Experimental Psychology: Learning, Memory, and Cognition, 36(6), 1466-1479. doi:10.1037/ a0020851

Mandler, G. (1980). Recognizing: The judgment of previous occurrence. Psychological Review, 87(3), 252-271. doi:10.1037/0033-295X.87. 3.252

Meng, X. L., Rosenthal, R., \& Rubin, D. B. (1992). Comparing correlated correlation coefficients. Psychological Bulletin, 111(1), 172-175. doi:10.1037/0033-2909.111.1.172

Miller, G. A. (1956). The magical number seven plus or minus two: Some limits on our capacity for processing information. Psychological Review, 63(2), 81-97.

Morey, R. D. (2008). Confidence intervals from normalized data: A correction to Cousineau (2005). Tutorial in Quantitative Methods for Psychology, 4(2), 61-64.

Morey, R. D., Morey, C. C., Brisson, B., \& Tremblay, S. (2012). A critical evaluation of $\mathrm{c}$ as a measure of mnemonic resolution. Journal of Experimental Psychology: Human Perception and Performance, 38(4), 1069-1072. doi:10.1037/a0027335

Paivio, A., \& Bleasdale, F. (1974). Visual short-term memory: A methodological caveat. Canadian Journal of Psychology/Revue Canadienne De Psychologie, 28(1), 24-31. doi:10.1037/h0081973

Parks, C. M., \& Yonelinas, A. P. (2007). Moving beyond pure signaldetection models: Comment on Wixted (2007). Psychological Review, 114(1), 188-201. doi:10.1037/0033-295X.114.1.188

Peich, M.-C., Husain, M., \& Bays, P. M. (2013). Age-related decline of precision and binding in visual working memory. Psychology and Aging, 28(3), 729-743. doi:10.1037/a0033236

Phillips, W. A. (1974). On the distinction between sensory storage and short-term visual memory. Perception \& Psychophysics, 16(2), 283-290.

Pitt, M. A., \& Myung, I. J. (2002). When a good fit can be bad. Trends in Cognitive Sciences, 6(10), 421-425. doi:10.1016/S1364-6613(02) 01964-2

Pratte, M. S., Park, Y. E., Rademaker, R. L., \& Tong, F. (2017). Accounting for stimulus-specific variation in precision reveals a discrete capacity limit in visual working memory. Journal of Experimental Psychology: Human Perception and Performance, 43(1), 6-17. doi:10.1037/xhp0000302

Roberts, S., \& Pashler, H. (2000). How persuasive is a good fit? A comment on theory testing. Psychological Review, 107(2), 358-367. 
Roberts, S., \& Pashler, H. (2002). Reply to Roders and Rowe (2002). Psychological Review, 109(3), 605-607. doi:10.1037//0033-295X. 109.3.605.

Rodgers, J. L., \& Rowe, D. C. (2002a). Postscript: Theory development should not end (but always begins) with good empirical fits: Response to Roberts and Pashler's (2002) reply. Psychological Review, 109(3), 603-604. doi:10.1037/h0087895

Rodgers, J. L., \& Rowe, D. C. (2002b). Theory development should begin (but not end) with good empirical fits: A comment on Roberts and Pashler (2000). Psychological Review, 109(3), 599604. doi:10.1037//0033-295X.109.3.599

Rouder, J. N., Morey, R. D., Cowan, N., Zwilling, C. E., Morey, C. C., \& Pratte, M. S. (2008). An assessment of fixed-capacity models of visual working memory. Proceedings of the National Academy of Sciences of the United States of America, 105(16), 5975-5979. doi: 10.1073/pnas.0711295105

Rouder, J. N., Morey, R. D., Morey, C. C., \& Cowan, N. (2011). How to measure working memory capacity in the change detection paradigm. Psychonomic Bulletin \& Review, 18(2), 324-330. doi:10. 3758/s13423-011-0055-3

Scolari, M., Vogel, E. K., \& Awh, E. (2008). Perceptual expertise enhances the resolution but not the number of representations in working memory. Psychonomic Bulletin \& Review, 15(1), 215-222. doi: 10.3758/PBR.15.1.215

Sherman, S. J., Atri, A., Hasselmo, M. E., Stern, C. E., \& Howard, M. W. (2003). Scopolamine impairs human recognition memory: Data and modeling. Behavioral Neuroscience, 117(3), 526-539. doi:10.1037/ 0735-7044.117.3.526

Smith, E. E., \& Nielsen, G. D. (1970). Representations and retrieval processes in short-term memory: Recognition and recall of faces. Journal of Experimental Psychology, 85(3), 397-405. doi:10. 1037/h0029727

Spachtholz, P., Kuhbandner, C., \& Pekrun, R. (2014). Negative affect improves the quality of memories: Trading capacity for precision in sensory and working memory. Journal of Experimental Psychology: General, 143(4), 1450-1456. doi:10.1037/ xge0000012

Tulving, E. (1985). Memory and consciousness. Canadian Psychology/ Psychologie Canadienne, 26(1), 1-12. doi:10.1037/h0080017

van den Berg, R., Awh, E., \& Ma, W. J. (2014). Factorial comparison of working memory models. Psychological Review, 121(1), 124-149. doi:10.1037/a0035234

van den Berg, R., Shin, H., Chou, W.-C., George, R., \& Ma, W. J. (2012). Variability in encoding precision accounts for visual short-term memory limitations. Proceedings of the National Academy of
Sciences of the United States of America, 109(22), 8780-8785. doi:10.1073/pnas.1117465109

Vogel, E. K., Woodman, G. F., \& Luck, S. J. (2006). The time course of consolidation in visual working memory. Journal of Experimental Psychology: Human Perception and Performance, 32(6), 14361451. doi:10.1037/0096-1523.32.6.1436

Wee, N., Asplund, C. L., \& Chee, M. W. L. (2013). Sleep deprivation accelerates delay-related loss of visual short-term memories without affecting precision. Sleep, 36(6), 849-856. doi:10.5665/sleep.2710

Wickens, T. D. (2001). Elementary signal detection theory. New York, NY: Oxford University Press.

Wilken, P., \& Ma, W. J. (2004). A detection theory account of change detection. Journal of Vision, 4(12), 1120-1135. doi:10.1167/4.12.11

Wixted, J. T. (2007). Dual-process theory and signal-detection theory of recognition memory. Psychological Review, 114(1), 152-176. doi: 10.1037/0033-295X.114.1.152

Xie, W., \& Zhang, W. (2016). Negative emotion boosts quality of visual working memory representation. Emotion, 16(5), 760-774. doi:10. 1037/emo0000159

Xie, W., \& Zhang, W. (2017a). Discrete item-based and continuous configural representations in visual short-term memory. Visual Cognition, 1-13. doi:10.1080/13506285.2017.1339157

Xie, W., \& Zhang, W. (2017b). Familiarity increases the number of remembered Pokémon in visual short-term memory. Memory \& Cognition, 45(4), 677-689. doi:10.3758/s13421-016-0679-7

Xie, W., \& Zhang, W. (2017c). Negative emotion enhances mnemonic precision and subjective feelings of remembering in visual longterm memory. Cognition, 166, 73-83. doi:10.1016/j.cognition. 2017.05.025

Ye, C., Zhang, L., Liu, T., Li, H., \& Liu, Q. (2014). Visual working memory capacity for color is independent of representation resolution. PLoS ONE, 9(3), e91681-e91686. doi:10.1371/journal.pone. 0091681

Yonelinas, A. P., \& Parks, C. M. (2007). Receiver operating characteristics (ROCs) in recognition memory: A review. Psychological Bulletin, 133(5), 800-832. doi:10.1037/0033-2909.133.5.800

Zhang, W., \& Luck, S. J. (2008). Discrete fixed-resolution representations in visual working memory. Nature, 453(7192), 233-235. doi:10. 1038/nature06860

Zhang, W., \& Luck, S. J. (2009). Sudden death and gradual decay in visual working memory. Psychological Science, 20(4), 423-428. doi:10.1111/j.1467-9280.2009.02322.x

Zhang, W., \& Luck, S. J. (2011). The number and quality of representations in working memory. Psychological Science, 22(11), 1434 1441. doi:10.1177/0956797611417006 